\title{
Méthode nouvelle de recherche des larves filariformes de Strongyloides stercoralis dans les coprocultures
}

\author{
par M. KREMER
}

\begin{abstract}
A notre connaissance peu d'études ont été faites depuis Fülleborn (1) sur le phototropisme et le thermotropisme des larves de Strongyloides stercoralis. Selon cet auteur, les larves filariformes seraient phototropiques négatives ou indifférentes et thermotropiques positives. Les résultats que nous avons obtenus ne sont pas aussi tranchés et leur application à la recherche de larves dans les coprocultures doit faire intervenir plusieurs mécanismes, que nous exposerons en premier, sommairement.

Nos études sur les formes adultes stercoraires et larves rhabditiformes sont encore trop fragmentaires pour entrer dans cette étude. Notons cependant qu'il ne semble pas $\mathrm{y}$ avoir pour elles de phototropisme positif net.
\end{abstract}

\section{Méthode d'étude des tropismes}

Les larves ont été obtenues à partir de selles de divers malades. Les formes filariformes ont été « cultivées » selon la méthode classique du mélange de matières fécales, de noir animal et d'eau. Le mélange est bien remué de façon à prendre un aspect relativement homogène et est placé sur plusieurs épaisseurs de papier filtre dans des boîtes de Pétri. Après 48 heures de conservation à une température de $25^{\circ}$ environ, nous obtenons généralement des formes filariformes assez nombreuses.

Les larves ont été étudiées dans les conditions suivantes:

a) dans leur milieu de culture ;

b) dans le milieu de culture immergé ;

c) dans l'eau pure.

Le comportement des larves était observé au binoculaire à l'éclairage direct, le spot lumineux servant de source lumineuse. Le déplacement de la source lumineuse se

(1) Nous adressons nos sincères remerciements à M. le Professeur Roman, qui a bien voulu nous communiquer des références bibliographiques concernant ce sujet. 
fait par déplacement du milieu où évoluent les larves, le spot lumineux restant fixe. On peut également déplacer légèrement le spot lumineux dans les limites du champ d'observation du binoculaire.

Le plus grand inconvénient de ce mode d'observation est la difficulté de séparer ce qui revient à l'action de la lumière et ce qui est dû aux augmentations locales de température sous l'effet de l'éclairage.

Nous avons essayé d'étudier la composante chaleur à part. Pour cela nous avons placé dans les milieux de culture de petits tubes contenant de l'eau chaude, à des températures diverses, mais, par de rapides éclairages, nous n'avons pas constaté de rassemblement de larves autour de ces sources de chaleur très localisées. Ces résultats qui semblent en contradiction avec ceux de Fülleborn sont peut être dus à une insuffisance de notre méthode.

\section{COMPORTEMENT DES LARVES FILARIFORMES SOUS LE SPOT LUMINEUX.}

Précisons d'emblée, après ce que nous venons d'exposer, que nous n'éliminons pas définitivement un thermotropisme positif des larves, mais comme il ressort des expériences suivantes, il semble bien y avoir également un certain phototropisme.

L'effet excitatoire de la lumière, phénomène bien connu, est très net. Les larves placées dans le spot lumineux sont de plus en plus agitées. Mais on observe également une concentration de plus en plus grande des larves dans le spot lumineux. Ce phénomène est encore plus net, si par un réglage de la tache lumineuse on crée un certain gradient de luminosité. La plupart des larves du milieu tendent à se rassembler sous la partie la plus intense du spot. Nous avons obtenu les mêmes résultats, dans le milieu pâteux des cultures, dans les milieux semi-liquides des cultures immergées et sur les larves placées dans l'eau pure. Dans le milieu pâteux, le phénomène est cependant assez rapidement interrompu, dès que la chaleur du spot lumineux a entraîné une certaine dessiccation locale. Dans les milieux liquides, le phénomène semble s'épuiser après des temps assez longs, de plusieurs dizaines de minutes, pour reprendre aussi nettement après un certain temps de repos.

Nous avons essayé d'étudier le déplacement des larves grâce à l'attraction produite par le spot lumineux. En faisant se déplacer le spot lumineux dans les faibles limites du champ du binoculaire, nous avons constaté très nettement que les larves suivaient fidèlement son point d'impact. Par déplacement du milieu de culture, permettant de bien plus grands mouvements, nous remarquons que les larves restent constamment (pour peu que le mouvement ne soit pas trop rapide) au même endroit, sous l'impact du spot, escaladant les « pics » et traversant les « mares et les creux » du milieu de culture. Le même résultat est obtenu dans les milieux liquides, où les larves semblent nager sur place. On peut ainsi déplacer le milieu à une vitesse de 2 à $4 \mathrm{~cm}$ à la minute, qui doit correspondre à la vitesse de déplacement des larves. Ce phénomène, lorsque les larves sont nômbreuses, permet de faire voyager à travers toute la préparation un important ruban de larves, visible à l'œil nu. 


\section{Application à la recherche des larves dans les coprocultures}

Par la méthode classique de la coproculture, il est en général aisé de faire le diagnostic d'anguillulose. Le tropisme exercé par le spot lumineux présente un intérêt dans les cultures pauvres et négatives, car il permet de s'assurer de l'absence ou de la présence de larves, même si elles sont rares.

Le procédé consistant à attirer les larves dans des cônes de papier filtre remplis d'eau est bien connu. Celui que nous employons est encore plus simple. Le milieu de culture, légèrement couvert d'eau, est placé sous le spot lumineux du binoculaire, réglé de façon à créer un certain gradient lumineux. Le maximum de luminosité correspondant au champ de vision du binoculaire. On laisse le dispositif en place pendant une dizaine de minutes, après quoi on retrouve dans le champ de vision la plupart des larves existant dans la préparation. Si le spot lumineux n'est pas réglable de façon à créer un gradient, le fait de recouvrir la préparation d'eau en tiendra lieu dans une certaine mesure.

Nous avons réalisé l'expérience suivante, prouvant l'efficacité de la méthode. Des larves en petit nombre et bien défini ont été placées dans des milieux neufs ou dans l'eau pure. Nous avons pu sans trop de difficultés les récupérer toutes en procédant avec la méthode précédemment décrite. Ce procédé peut donc servir aussi bien à recueillir après les avoir concentrées en un point de nombreuses larves, ou à les détecter lorsqu'elles sont rares.

\section{Conclusion}

Nous indiquons un procédé simple pour recueillir et trouver les larves filariformes de Strongyloides stercoralis, obtenues par coproculture classique. Les tropismes intervenant dans ce procédé sont étudiés, mais ils ne permettent pas encore de conclusion formelle, si ce n'est que les résultats admis jusqu'à présent doivent être réexaminés.

(Institut de Parasitologie de la Faculté de Médecine de Strasbourg [Bas-Rhin, France] [Directeur: Professeur J. CaLlot])

\section{Bibliographie}

Fülleborn (Fr.), 1924. - Ueber « Taxis » (Tropismus) bei Strongyloides und Ankylostomenlarven. Arch. f. Schifs-u.-Tropen-Hyg., 28, 144-165.

Kosuge (I.), 1924. - Wie weit wirken bei dem Eindringen von Strongyloides larven und anderen parasitischen Nematoden in das Gewebe spezifische Reize ? Arch. f. Schifsu.-Tropen-Hyg., 28, 179-187.

Langeron (M.), 1942. - Précis de Microscopie, 6 Edition, Paris.

Annales de Parasitologie humaine et comparée (Paris), t. 40, 1965, $\mathrm{n}^{\circ} 2$ 
Roman (E.), 1951. - Etude écologique et morphologique sur les Acanthocéphales et les Nématodes parasites des rats de la région lyonnaise. Mém. Mus. Nat. Hist. Nat., Nlle série, A, Zool., 2, 49-270.

Roman (E.), 1955. - Comportement des stades libres de Strongyloides ratti vis-à-vis de quel- : ques variations du milieu. Rev. Iber. Parasit., tome extraordinaire, mars, 397-410. 